

\title{
LA AUTOESTIMA Y LOS ESTILOS DE APRENDIZAJE EN RELACIÓN CON EL PROMEDIO ACADÉMICO EN EL NIVEL MEDIO SUPERIOR
}

\author{
SELF-ESTEEM AND LEARNING STYLES IN RELATION \\ TO THE ACADEMIC AVERAGE IN HIGH SCHOOL
}

\section{DIANA GLORIA GARCÍA COLIÓN}

\section{RESUMEN}

La presente investigación tiene como objetivo identificar el nivel de autoestima general, social, escolar, del hogar y total de los alumnos de nivel Medio Superior y los estilos de aprendizaje de estos, para determinar si existe una relación entre ellos. Se utilizó una investigación de tipo cuantitativo para obtener resultados de manera objetiva. Los instrumentos utilizados para recolectar la información fueron el Inventario de los Estilos de Aprendizaje de Honey-Alonso (CHAEA) y el Inventario de Autoestima de Coopersmith forma A (adaptado en Chile por Brinkmann, Segure y Solar 1989). Para analizar los resultados se utilizaron los programas IBM SPSS y el SPC XL, ambos versión de prueba, y para medir el grado de relación entre las variables se basó en el coeficiente de Pearson. Los resultados indican que los jóvenes que tienen un nivel de autoestima alto o muy alto tienden a utilizar un estilo de aprendizaje reflexivo o pragmático, los cuales requieren de procesos más complejos cognitivamente. Además, la autoestima total de los estudiantes está mayormente influenciada por los aspectos sociales, es decir, por la autoestima escolar, familiar y social.

PALABRAS CLAVE: APRENDIZAJE, PROMEDIO, AUTOESTIMA, ALUMNOS, MOTIVACIÓN, VARIABLE.

\section{ABSTRACT}

The objective of this research is to identify the level of general, social, school, home and total self-esteem, and the learning styles in high school students, and to determine if there is a relationship between them. Quantitative research was used to obtain results in an objective manner. The instruments used to collect the information were the Inventory of Learning Styles of Honey-Alonso (CHAEA) and the Inventory of Self-esteem of Coopersmith form A (adapted in Chile by Brinkmann, Segure and Solar 1989). To analyze the results, the IBM SPSS and the SPC XL programs were used, both test version, and to measure the degree of relationship between the variables was based on the Pearson coefficient. The results indicate that young people who have a high or very high level of self-esteem tend to use a reflective or pragmatic learning style, which requires more complex cognitive processes. In addition, the students' total self-esteem is mostly influenced by social aspects, that is, by school, family and social self-esteem.

KEYWORDS: LEARNING, AVERAGE, SELF-ESTEEM, STUDENTS, MOTIVATION, VARIABLE. 


\section{ANTECEDENTES}

- $n$ la actualidad, ante un marco globalizado, la educación busca que los alumnos tengan un alto desempeño académico y que los estudiantes logren adquirir y desarrollar las habilidades necesarias para la vida. En este proceso, y bajo la nueva Reforma Integral de la Educación Media Superior en México, se requiere considerar las diferencias individuales de los jóvenes para que el docente diseñe correctamente las estrategias de enseñanza considerando los diferentes estilos de aprendizaje que tiene cada persona. Sin embargo, en la realidad se tienen casos donde los estilos de aprendizaje utilizados por los estudiantes son ineficaces y, al profundizar sobre las causas, se encuentra que se deben a una serie de acontecimientos pasados que afectaron de manera negativa en su desarrollo psicológico enfocado en la autoestima. Por lo tanto, como docentes es importante conocer a los alumnos y sus necesidades para poder coadyuvar con su desarrollo integral.

Con el aprendizaje formal 0 informal se adquieren habilidades, conocimientos, valores, actitudes y reacciones emocionales. Los cambios se atribuyen a la experiencia, es decir, a los acontecimientos de la vida del aprendiz y que, desde el punto de vista cognitivo, se relaciona con el proceso de pensamiento. Dentro de este proceso de aprendizaje, la metacognición, que se desarrolla a partir de la adolescencia, es quien guía el proceso de información y supervisa la eficacia de las diferentes estrategias que el aprendiz aplica en una tarea cualquiera, es decir, piensa sobre el pensamiento (Ormrod, 2008).
Aquí surge que cada persona cuenta con estrategias internas propias que le permiten afrontar su realidad generando así las diferencias individuales dentro de un salón de clases que se relacionan directamente con el desempeño académico.

Las diferencias individuales están influidas por los cambios biológicos que sufre el adolescente y por el contexto social donde él se desarrolla. Bronfenbrenner (1989) propuso el Modelo Bioecológico del Desarrollo Humano, y los principales sistemas donde el ser humano se desarrolla son: la familia, el ambiente religioso, el salón de clase y el grupo de pares. El más importante es la familia, debido a que su influencia inicia desde el nacimiento y ésta depende de su estructura familiar y los estilos de crianza que los padres tomen para educar a sus hijos (Woolfolk, 2010). La mezcla de estas experiencias en los jóvenes afecta tanto positiva como negativamente su desarrollo psicológico que después repercute en su autoestima.

La autoestima es una reacción afectiva: es el sentido general de valía y bienestar de la persona. Los términos que se relacionan con ella son la ima- gen personal, el concepto personal y la autopercepción. Los niños al llegar a la adolescencia mejoran su toma de perspectiva, su capacidad de entender los pensamientos y sentimientos de los demás debido a que el desarrollo cognitivo funciona como un núcleo organizacional que afecta todas las áreas del pensamiento. Este pensamiento abstracto los conlleva a plantearse preguntas sobre cuáles son sus características que los hacen ser quiénes son, cómo los perciben otras personas, entre otras (Arnett, 2008). Todo esto cambia su autoestima y modifica su identidad, es decir, la percepción de sus capacidades y características (Arnett, 2008, p.164). Debido a que esto se relaciona directamente con el bienestar de la persona, su nivel de autoestima influye en sus acciones y sus relaciones sociales en el salón de clases como lo son en el trabajo colaborativo.

La identidad se desarrolla a lo largo de la vida y, a diferencia de la autoestima, es una imagen consistente de sí mismo. Erikson planteó la teoría de las Etapas del Desarrollo Psicosocial, en donde el ser humano pasa por etapas interdependientes con sus metas, preocupaciones, logros y riesgos específicos, así como sus
La metacognición es quien guía el proceso de información y supervisa la eficacia de las diferentes estrategias que el aprendiz aplica en una tarea cualquiera, es decir, piensa sobre el pensamiento (Ormrod, 2008) 
alternativas positivas y potencialmente dañinas. En el aspecto de la educación formal, durante el preescolar los conflictos principales son la confianza, autonomía e iniciativa, seguidos de la laboriosidad contra la inferioridad en la primaria y secundaria y, posteriormente, al llegar al nivel medio superior, el conflicto de la identidad contra la confusión de roles (Woolfolk, 2010).

Las acciones tomadas ante estos conflictos se trasladan al desempeño escolar y se pueden observar, por ejemplo, al momento de realizar actividades. Es decir, se pueden encontrar a personas que realizan la actividad con alto nivel de confianza y lo llevan a su revisión una vez terminado, mientras que otras personas lo realizan por partes tomando una pausa para preguntar al profesor si lo está haciendo correctamente y así continuar con ella.

La metacognición trasladada a la educación formal está formada por conocimientos y habilidades que le permiten a la persona identificar sus propias capacidades de aprendizaje y de memoria, así como las tareas que puede ejecutar de una manera realista. Además, le permiten saber qué estrategias le son efectivas y planear las tareas para que pueda lograrlas con éxito y, sobre todo, que pueda recuperar esa información almacenada previamente.

De aquí surge el término Autorregulación: cuando el estudiante empieza a regular el propio aprendizaje estableciendo sus objetivos y planeándolos, automotivándose, controlando su atención, aplicando estrategias de aprendizaje, autocontrolándose

\section{La metacognición trasladada a la educación formal está formada por conocimientos y habilidades que le permiten a la persona identificar sus propias capacidades de aprendizaje y de memoria, así como las tareas que puede ejecutar de una manera realista}

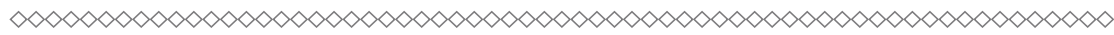

$y$, principalmente, autoevaluándose (Ormrod, 2008). Los problemas en el desempeño escolar surgen, por ejemplo, cuando la persona no tiene valía y se siente incapaz de poder lograr una tarea asignada 0 no encuentra motivación para hacerlo.

La motivación es un aspecto importante, puesto que influye en la conducta llevando a la persona a realizar ciertos actos para conseguir un determinado fin. Los dos principales tipos de motivación son la cognitiva y la social. La primera se relaciona con el interior de la persona, es decir, de sus estados internos mentales; y la segunda es la que se presenta en el ámbito escolar donde la relación con los pares, profesores y los padres son los que determinan la motivación del alumno. Sin embargo, en esta última es importante que dicha relación genere una motivación intrínseca para el alumno, es decir, que no dependa de una recompensa externa para que realice cierta acción (García, 2011). Como se mencionó anteriormente, el contexto familiar influye en los adolescentes al grado de afectar en su motivación, por otro lado, el rol del profesor también es punto clave para mejorar dicha motivación y su respectivo rendimiento escolar.

Los estilos de enseñanza son la forma personal que cada profesor tiene en su actuar dentro del salón de clases y consta de sus actitudes, aptitudes, potencialidades y debilidades para determinar los estilos de aprendizaje de sus estudiantes y poder desarrollar un ambiente instruccional óptimo (Pinelo, 2008). Para García y Pascual (1994, citados por López, 1996) lo que se busca con los estilos de enseñanza en la actualidad es mejorar el rendimiento de los alumnos desarrollando habilidades y estrategias para lograr el autoconocimiento y conciencia del estudiante sobre sus propias competencias, enfoques y estilos de aprendizaje.

Los estilos de aprendizaje, con base en Cazau (2001, citado por González, 2011), son los rasgos cognitivos, afectivos y fisiológicos que sirven como indicadores relativamente estables de cómo los alumnos perci- 


\section{TABLA 1. Resultados de la Media y Desviación Estándar de la Autoestima}

\begin{tabular}{|c|c|c|c|c|}
\hline AUTOESTIMA & $X$ & S.D. & LÍMITE INFERIOR & LÍMITE SUPERIOR \\
\hline General & 42.23 & 15.76 & 20 & 75 \\
\hline Social & 50.56 & 9.25 & 21 & 70 \\
\hline Escolar & 61.03 & 11.99 & 24 & 78 \\
\hline Hogar & 49.31 & 7.56 & 28 & 70 \\
\hline Total & 56.06 & 15.32 & 20 & 80 \\
\hline
\end{tabular}

ben interacciones y responden a sus ambientes de aprendizaje. Todo esto es construido en la experiencia sociohistórica y le permiten adaptarse. Éstos se pueden imitar de una persona a otra siempre y cuando sea de manera positiva, y no está limitado a aplicar sólo un estilo sino que puede emplear combinaciones de acuerdo a sus intereses (González, 2011).

La institución donde se llevó a cabo la investigación posee un alto índice de deserción escolar, ya que durante el 1er semestre los grupos tienen apróximadamente 50 alumnos y, después de un año, tienen un promedio de 35 alumnos; un 30\% de los alumnos no continúan con sus estudios. Dentro de las causas principales es la reprobación de materias que, de acuerdo a las entrevistas que el departamento de Servicios Escolares ha realizado, se debe a los estilos de aprendizaje que cada alumno posee.

A través del programa de Tutorías se detectaron jóvenes con problemas como falta de confianza, inseguridad en su físico o viven en familias disfuncionales, por lo que su rendimiento y promedio académico es regular 0 bajo. En estos grupos hay jóvenes con alta confianza, seguridad en su físico, familias funcionales y tradicionales, y con alto rendimiento y promedio académico. Esto permite analizar el impacto de su autoestima con los estilos de aprendizaje que conllevan a un promedio académico alto.

Con base en lo anterior, surge la importancia de conocer el impacto de la autoestima de los jóvenes en relación con los estilos de aprendizaje. El presente trabajo pretende responder y aportar información a la comunidad educativa en relación a la pregunta: ¿Cómo impacta la autoestima en la aplicación de los estilos de aprendizaje en relación con el promedio académico? Los antecedentes descritos en párrafos anteriores entorno a las diferencias individuales, la autoestima y los estilos de aprendizaje dan sustento al planteamiento del problema de la presente investigación.

El principal objetivo de esta investigación es conocer el impacto de la autoestima con relación a la aplicación de los estilos de aprendizaje y el promedio académico de los estudiantes de la especialidad de Elec- trónica y Laboratorista en una Institución Técnica ubicada en la ciudad de Guadalupe en Nuevo León.

La hipótesis planteada para la presente investigación establece que la autoestima impacta directamente en la selección de los estilos de aprendizaje de los jóvenes y, por consiguiente, en su promedio académico, es decir, las personas con alta autoestima elegirán estilos de aprendizaje más complejos y, a su vez, tendrán un promedio escolar más alto.

La presente investigación buscó encontrar la correspondencia entre la autoestima y los estilos de aprendizaje en relación con el promedio académico, por lo que se optó en utilizar un método cuantitativo. Se realizó una investigación no experimental, o ex-post-facto, la cual es referida a la observación de la situación una vez ocurrido el fenómeno y no existe manipulación de las variables (Ortíz, 2006). Para la presente investigación, la variable independiente fue la autoestima y la variable dependiente los estilos de aprendizaje de los estudiantes. El diseño fue transversal con una metodología cuantitativa. 
Los participantes del estudio fueron $50 \%$ estudiantes de la especialidad de Laboratorista y $50 \%$ de la especialidad de Electrónica. La muestra fue de 90 estudiantes, $50 \%$ de la especialidad de Electrónica y $50 \%$ de la especialidad de Laboratorista.

El instrumento utilizado para la medición de la autoestima fue el Inventario de Autoestima de Coopersmith, que se obtuvo (versión en español) del estudio realizado por Brinkmann, Segure y Solar (1989). Se transcribió a un documento de Word que se entregó físicamente a los estudiantes.

El promedio académico se obtuvo de los acumulados a lo largo de la carrera técnica tipificándose como:

- Regularcon 6

- Bueno con 7-8

- MuyBueno con 9

- Excelente con 10

Dichos datos se obtuvieron del Sistema de Servicios Escolares de la Educación Media Superior, el cual contiene la base de datos de las calificaciones de los estudiantes.

El instrumento que se utilizó para recabar información sobre los estilos de aprendizaje de los alumnos fue el Cuestionario Honey-Alonso de Estilos de Aprendizaje (CHAEA), el cual es una adaptación del LSQ de Honey y Mumford llevado a cabo por la Dra. Catalina M. Alonso (Salas, 2008). La aplicación se llevó a cabo en el laboratorio de computación para los jóvenes de la especialidad de Laboratorista y en el taller de PLC para los estudiantes de la especialidad de Electrónica. Realizado vía internet durante las horas de Tutorías, los estudiantes ingresaron a la página: http://www.estilosdeaprendizaje.es/ chaea/chaea.htm (Cué, 2014).

Se utilizaron los programas estadísticos IBM SPSS versión de prueba y el SPC XL versión de prueba. De la base de datos general se capturaron las autoestimas, estilos de aprendizaje, sexo, edad y promedio académico, y luego se procedió a obtener la matriz de correlación de Pearson. Así mismo, se obtuvo para cada uno de los datos la media y desviación estándar. Posteriormente, se realizó una tipificación para cada estilo de aprendizaje y nivel de autoestima quedando de la siguiente manera: Muy Bajo, Bajo, Moderado, Alto y Muy Alto. Y con la ayuda de una tabla dinámica del programa de Excel se sacaron los porcentajes de preferencia, así como los porcentajes por género. Por último, los resultados obtenidos se contrastaron con los estudios realizados previamente y con la teoría que sustenta la investigación.

\section{RESULTADOS}

La Autoestima General está 8 puntos debajo de la media, sin embargo, dentro del rango de la desviación estándar se considera que la media está dentro de los parámetros normales. La desviación estándar obtenida es de 15.76 puntos, por lo que se tienen jóvenes que alcanzaron 26 puntos, los cuales se ubican en Bajo y Muy Bajo nivel (Tabla 1, pág. 30).

La Autoestima Social se ubicó, tanto para la media como para la desviación estándar, en el mismo nivel que los resultados obtenidos por Coopersmith. Por lo que se puede concluir que los jóvenes cuentan con un nivel Normal en esta escala. La Autoestima Escolar estuvo 11 puntos por encima de lo definido por Coopersmith con una desviación estándar de 12 puntos, lo cual significa que los jóvenes

TABLA 2. Resultados de la Autoestima por Tipificación y Porcentajes

\begin{tabular}{|c|c|c|c|c|c|}
\hline NIVEL & $\begin{array}{c}\text { AUTOESTIMA } \\
\text { GENERAL }\end{array}$ & $\begin{array}{c}\text { AUTOESTIMA } \\
\text { SOCIAL }\end{array}$ & $\begin{array}{c}\text { AUTOESTIMA } \\
\text { ESCOLAR }\end{array}$ & $\begin{array}{c}\text { AUTOESTIMA } \\
\text { HOGAR }\end{array}$ & $\begin{array}{c}\text { AUTOESTIMA } \\
\text { TOTAL }\end{array}$ \\
\hline Muy Bajo & $14 \%$ & $1 \%$ & $1 \%$ & $2 \%$ & $4 \%$ \\
\hline Bajo & $1 \%$ & $6 \%$ & $2 \%$ & $2 \%$ & $1 \%$ \\
\hline Normal & $77 \%$ & $68 \%$ & $34 \%$ & $87 \%$ & $44 \%$ \\
\hline Alto & $8 \%$ & $22 \%$ & $25 \%$ & $9 \%$ & $48 \%$ \\
\hline Muy Alto & $0 \%$ & $3 \%$ & $38 \%$ & $0 \%$ & $5 \%$ \\
\hline
\end{tabular}


TABLA 3. Resultados de Correlación de Pearson entre la Edad y las Autoestimas

\begin{tabular}{|c|c|c|c|c|c|}
\hline FACTOR & $\begin{array}{c}\text { AUTOESTIMA } \\
\text { GENERAL }\end{array}$ & $\begin{array}{c}\text { AUTOESTIMA } \\
\text { SOCIAL }\end{array}$ & $\begin{array}{c}\text { AUTOESTIMA } \\
\text { ESCOLAR }\end{array}$ & $\begin{array}{c}\text { AUTOESTIMA } \\
\text { HOGAR }\end{array}$ & $\begin{array}{c}\text { AUTOESTIMA } \\
\text { TOTAL }\end{array}$ \\
\hline Edad & -0.1581 & 0.1463 & 0.1266 & 0.0217 & 0.1423 \\
\hline
\end{tabular}

están en una escala dentro de Normal, Alto y Muy Alto nivel de autoestima en esta categoría.

La Autoestima del Hogar se encuentra dentro de la escala Normal establecida por Coopersmith, la única diferencia es que se tienen 3 puntos menos de desviación estándar. Se puede considerar que los jóvenes tienen una autoestima del hogar Normal. La Autoestima Total se encuentra 6 puntos por encima de la norma de Coopersmith, por lo que se considera dentro del nivel Normal. Además, a pesar de que se obtuvo una desviación estándar de 15 puntos y dado que la media fue superior, los resultados quedan dentro de los parámetros considerados normales.
Tipificando los resultados por escalas de nivel: Muy Bajo, Bajo, Normal, Alto y Muy Alto; se tienen los resultados mostrados en la Tabla 2 (pág. 31).

En la Autoestima General los porcentajes representativos se ubican en el nivel Normal con $77 \%$ y Muy Bajo con el $14 \%$, lo cual explica que la desviación estándar sea de 15.76 puntos. El $67 \%$ de la población femenina y el $60 \%$ de la población masculina se encuentra dentro de los parámetros normales. Mientras que el $20 \%$ de la población femenina y el $35 \%$ de la población masculina se encuentran en el nivel Muy Bajo.

En la Autoestima Social la media se encuentra en el nivel Normal con un
$68 \%$, pero también se tiene un nivel Alto con $22 \%$. La población femenina se divide en un $56 \%$ en el nivel Normal, $25 \%$ en el nivel Alto y un $11 \%$ en el nivel Muy Alto. Por otro lado, la población masculina está conformada por un $72 \%$ en el nivel Normal y $21 \%$ en el nivel Alto. En este último no hubo hombres que tuvieran Muy Alto nivel.

En la Autoestima Escolar se obtuvo generalmente un valor Alto. La población femenina se dividió en un $30 \%$ en el nivel Normal, $35 \%$ en el nivel Alto y $35 \%$ en el nivel Muy Alto. Por otro lado, en la población masculina el $40 \%$ estuvo en el nivel Muy Alto, el $22 \%$ en el Alto y $35 \%$ en el nivel Normal.

\section{GRÁFICA 1. Resultados de la Correlación de Pearson entre la Autoestima Total y las categorías de Coopersmith}

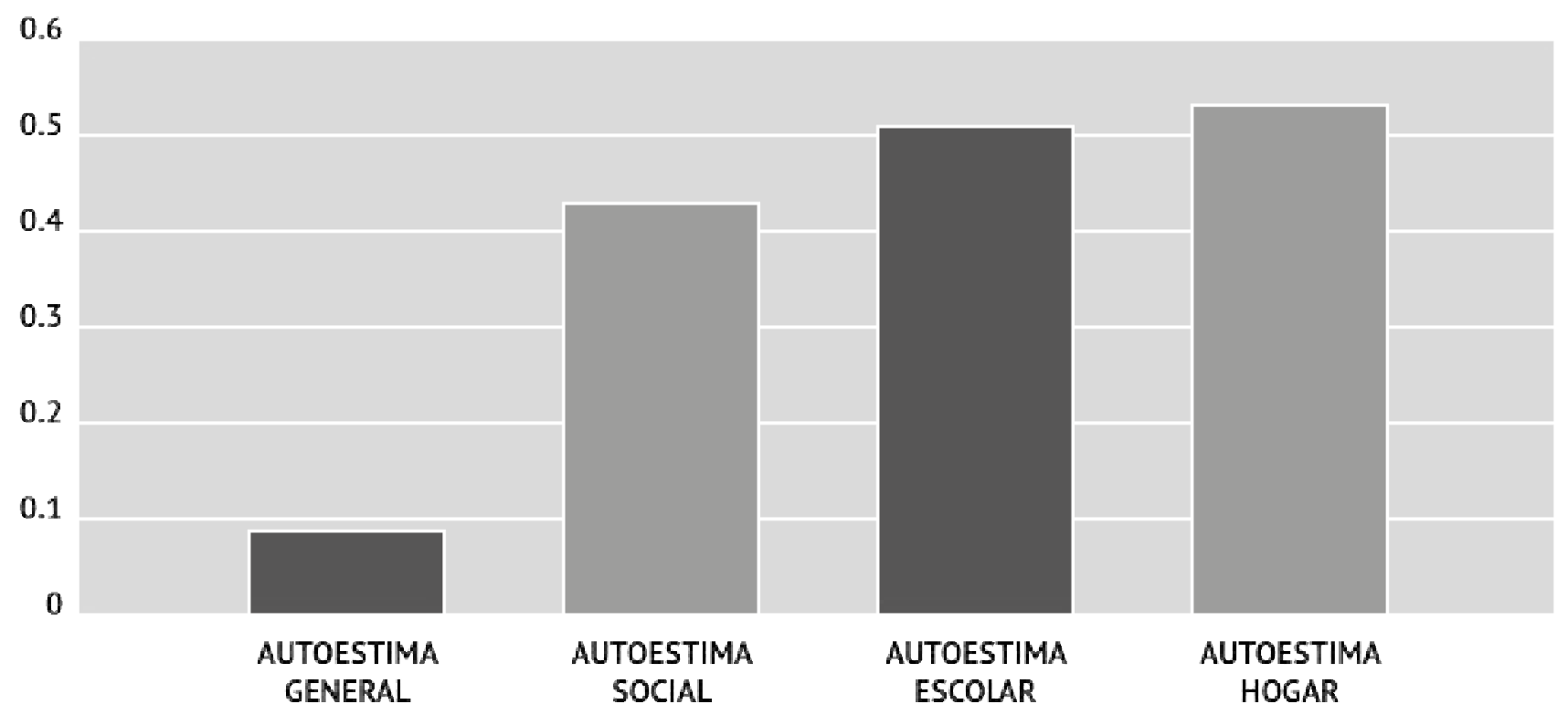

32 Presencia Universitaria 
TABLA 4. Resultados de la Media y Desviación Estándar de los Estilos de Aprendizaje

\begin{tabular}{|c|c|c|c|c|}
\hline $\begin{array}{c}\text { ESTILO DE } \\
\text { APRENDIZAJE }\end{array}$ & $\mathrm{X}$ & S.D. & LÍMITE INFERIOR & LÍMITE SUPERIOR \\
\hline Activo & 12.87 & 3.05 & 0 & 20 \\
\hline Reflexivo & 13.36 & 3.67 & 0 & 20 \\
\hline Teórico & 13.23 & 2.65 & 0 & 20 \\
\hline Pragmático & 14.05 & 2.87 & 0 & 20 \\
\hline
\end{tabular}

Para la Autoestima del Hogar, tanto la población femenina como masculina, tuvieron un $87 \%$ en el nivel Normal y $9 \%$ en el nivel Alto.

Por último, para la Autoestima Total se tiene que la media fue de un nivel Normal con tendencia a Alto. De aquí el $50 \%$ de la población femenina se ubica en un nivel Normal, seguido de un $25 \%$ en el nivel Alto y un $12 \%$ en el nivel Muy Alto. Por otro lado, en la población masculina el $55 \%$ se encontró en un nivel Alto y un $38 \%$ en el nivel Normal.
Arnett (2008) señala que el desarrollo de la adolescencia no depende de la edad sino de diferentes factores como el biológico, psicológico, social, entre otros. Para corroborarlo, se realizó el análisis correlacional obteniendo que los resultados dan valores muy cercanos al cero, por lo que se considera que no existe relación (Tabla 3, pág. 32).

Como 2do objetivo, se buscó identificar la o las categorías que impactan en la autoestima total y se tuvo como resultado dos porcentajes representativos: el $42 \%$ en el nivel Normal y $48 \%$ en el nivel Alto. Y con base a la tipificación, se encontró que dentro del nivel Normal el orden de relevancia fue primero para el Hogar, siguiente el General, después el Social y, por último, el Escolar. Caso contrario en el nivel Alto, ya que el más distintivo fue el Escolar, le continuó el Social y, con muy bajo impacto, el Hogar y General. Analizándose de manera global por medio del coeficiente de correlación de Pearson, se obtuvieron los resultados mostrados en la Gráfica 1 (pág. 32).

\section{GRÁFICA 2. Resultados de los Estilos de Aprendizaje por Tipificación y Porcentaje}

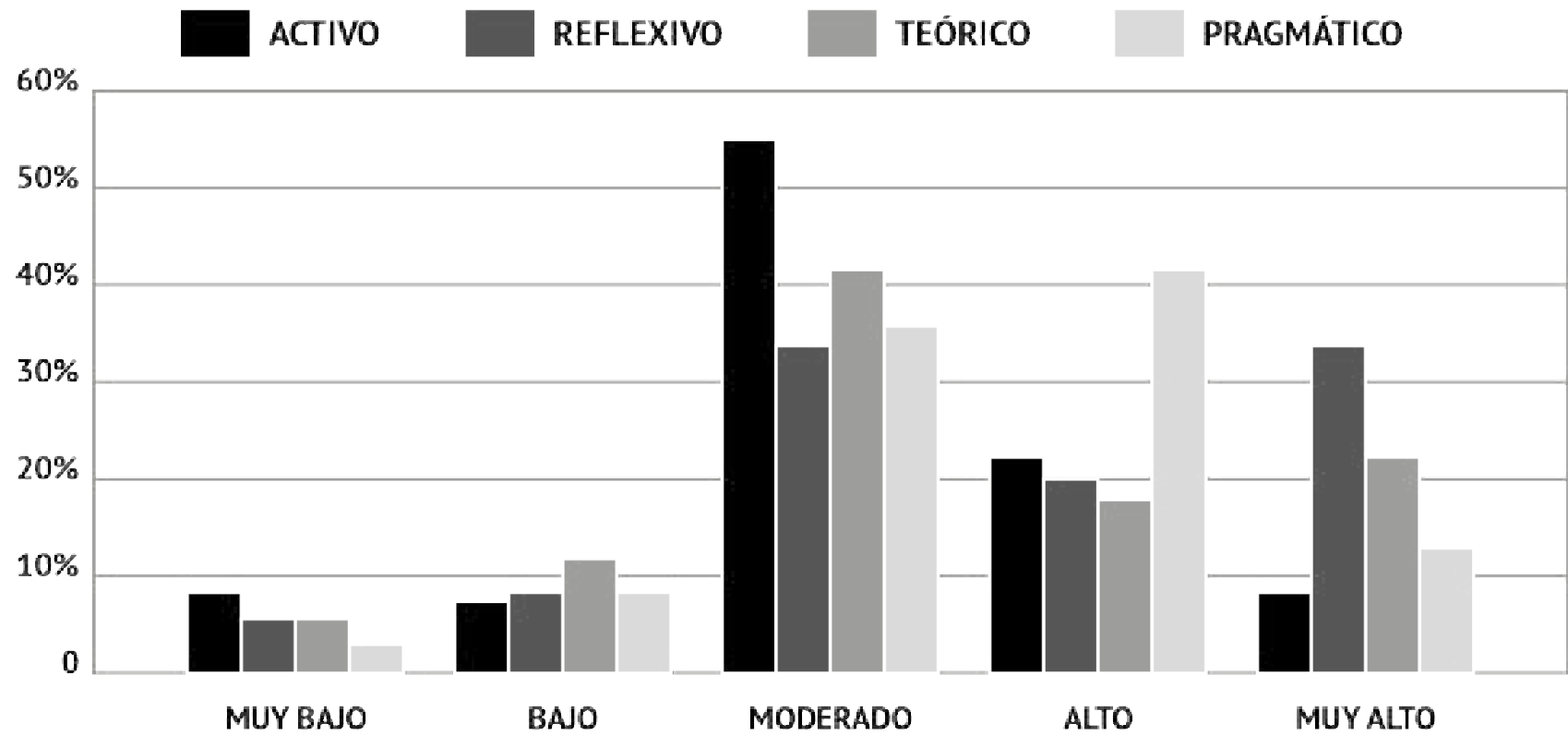


TABLA 5. Resultados del Coeficiente de Correlación de Pearson entre las Autoestimas y los Estilos de Aprendizaje

\begin{tabular}{|c|c|c|c|c|}
\hline AUTOESTIMA & $\begin{array}{c}\text { ESTILO } \\
\text { ACTIVO }\end{array}$ & $\begin{array}{c}\text { ESTILO } \\
\text { REFLEXIVO }\end{array}$ & $\begin{array}{c}\text { ESTILO } \\
\text { TEÓRICO }\end{array}$ & $\begin{array}{c}\text { ESTILO } \\
\text { PRAGMÁTICO }\end{array}$ \\
\hline General & -0.1333 & 0.0730 & -0.0150 & -0.097 \\
\hline Social & 0.2360 & 0.4358 & 0.2935 & 0.3328 \\
\hline Escolar & 0.2128 & 0.3964 & 0.0433 & 0.2081 \\
\hline Hogar & 0.2831 & 0.3563 & -0.0467 & 0.1821 \\
\hline Total & 0.5798 & 0.4234 & -0.1756 & 0.4642 \\
\hline
\end{tabular}

Como 3er objetivo, se propuso identificar los estilos de aprendizaje utilizados por los estudiantes, y se procedió a obtener la media para cada grupo así como su desviación estándar teniendo como resultado lo que se muestra en la Tabla 4 (pág. 33).

Tipificando los resultados por escalas de preferencia:

- Muy Bajo

- Bajo

- Moderado

- Alto

- MuyAlto

se obtuvieron los resultados que se muestran en la Gráfica 2 (pág. 33).

En el Aprendizaje Activo se desglosa que del $55 \%$ del nivel Moderado, el $32 \%$ fue seleccionado por la carrera de Electrónica mientras que el $23 \%$ fue seleccionado por los Laboratoristas. Del 22\% del nivel Alto, 15\% corresponde a los Laboratoristas y un $7 \%$ es para los Electrónicos.

En el Aprendizaje Reflexivo, el nivel Moderado y Muy Alto fueron seleccionados de igual manera por ambas carreras. La única distinción es en el aprendizaje Alto, donde el 13\% fue seleccionado por los Electrónicos y un $7 \%$ por los Laboratoristas.

En el Aprendizaje Teórico en el nivel Moderado un 25\% corresponde a la opción de los Electrónicos y un 17\% de los Laboratoristas, y en el nivel Muy Alto el $16 \%$ corresponde a Laboratoristas y un $7 \%$ a los Electrónicos.

Por último, en el Aprendizaje Pragmático para ambos niveles, Moderado y Alto, el porcentaje está compuesto equitativamente en las carreras, es decir, $17.5 \%$ para el nivel Moderado y $21 \%$ para el nivel Alto.
En el Estilo Activo se tuvo un 55\% en el nivel Moderado y un 22\% en el nivel Alto. El 55\% de los hombres y $53 \%$ de las mujeres optó por el nivel Moderado. Por otro lado, el 22\% de los hombres y $20 \%$ de las mujeres seleccionaron el nivel Alto. No hubo diferencias en cuanto al género.

En el Estilo Reflexivo los porcentajes relevantes fueron: el nivel Moderado con un 34\%, del cual corresponde un $40 \%$ de mujeres y un $31 \%$ de hombres; y el nivel Muy Alto con 33\%, donde corresponden el $35 \%$ de los hombres y un $27 \%$ de las mujeres.

El Estilo Teórico tuvo como principal el nivel Moderado, conformado por $44 \%$ de la población masculina y un $33 \%$ de la población femenina. En el Estilo Pragmático se tiene un $42 \%$ de preferencia Alta, donde lo eligió el $46 \%$ de la población masculina y un

TABLA 6. Resultados del Coeficiente de Correlación de Pearson entre el Promedio Académico y las Autoestimas

\begin{tabular}{|c|c|c|c|c|c|}
\hline FACTOR & $\begin{array}{c}\text { AUTOESTIMA } \\
\text { GENERAL }\end{array}$ & $\begin{array}{c}\text { AUTOESTIMA } \\
\text { SOCIAL }\end{array}$ & $\begin{array}{c}\text { AUTOESTIMA } \\
\text { ESCOLAR }\end{array}$ & $\begin{array}{c}\text { AUTOESTIMA } \\
\text { HOGAR }\end{array}$ & $\begin{array}{c}\text { AUTOESTIMA } \\
\text { TOTAL }\end{array}$ \\
\hline Edad & -0.1581 & 0.1463 & 0.1266 & 0.0217 & 0.1423 \\
\hline
\end{tabular}


$26 \%$ la población femenina. En caso contrario, la preferencia Moderada obtuvo el $40 \%$ de la población femenina y un $30 \%$ de la masculina.

Como 4to objetivo, se busca obtener la relación entre las diferentes categorías de autoestimas y los estilos de aprendizaje, dando como resultado los coeficientes de correlación de Pearson mostrados en la Tabla 5 (pág. 34). Se puede identificar que el Estilo Teórico es el que carece de relación con las autoestimas y, de igual manera, la Autoestima General en relación con los estilos de aprendizaje.

El 5to objetivo es relacionar las autoestimas con el promedio académico de los estudiantes, donde la media es 7.78 con una desviación estándar de 0.94 puntos (Tabla 6, pág. 34). Nuevamente, la Autoestima General carece de relación con el promedio académico. Y de manera global, la Autoestima Total tiene una correlación positiva significativa.

\section{CONCLUSIONES}

Tanto la autoestima como los estilos de aprendizaje tienen una correlación positiva con el promedio académico de tal manera que los jóvenes con un nivel bajo de autoestima utilizan estilos activos, los cuales se consideran que tienen menor autorregulación y un promedio académico bajo. En cambio, los que tienen un nivel alto de autoestima utilizan los estilos pragmáticos y reflexivos, los cuales se consideran en tener mayores procesos metacognitivos, ademas de tener un nivel alto de promedio académico.

Esto último comprueba la hipótesis planteada, la cual consiste en que las personas con alto nivel de autoestima eligen estilos de aprendizaje más complejos y, por consiguiente, tendrán mejor promedio académico.

A pesar de que se obtuvo como resultado que la autoestima general no impacta de manera significativa en la autoestima total de los jóvenes, no se puede dejar excluida dentro del desarrollo integral de los jóvenes; por lo que en la Institución Técnica se puede proponer dentro del programa de Tutorías actividades individuales y grupales que fomenten una autoestima personal positiva. Esto, a su vez, se apoya con las competencias genéricas establecidas dentro de la Reforma Integral de la Educación Media Superior donde señala que los jóvenes deben conocerse a sí mismos autovalorándose.

Los hallazgos obtenidos pueden rediseñar las asignaturas teniendo en cuenta procesos complejos que permitan un desarrollo metacognitivo en los jóvenes; así como sus actitudes hacia ellos en sentido de poder fomentar empatía e incrementar el nivel de autoestima. Por lo que será necesario realizar capacitación sobre el desarrollo del adolescente donde se expliquen los factores psicológicos influyentes y así los maestros puedan apoyar en el cambio del diseño de las materias para que exista menor resistencia. Así mismo, se podrán compartir con ellos estrategias que contribuyan al desarrollo positivo de la autoestima.

El estudio se realizó utilizando instrumentos confiables y válidos que, durante varios años, se han utilizado para llevar a cabo investigaciones relacionadas a la autoestima y los es- tilos de aprendizaje. Se han evaluado en diferentes ocasiones su validez de constructo y confiabilidad, resultando favorables. Es por esto que la validez interna de esta investigación se considera con una calidad adecuada.

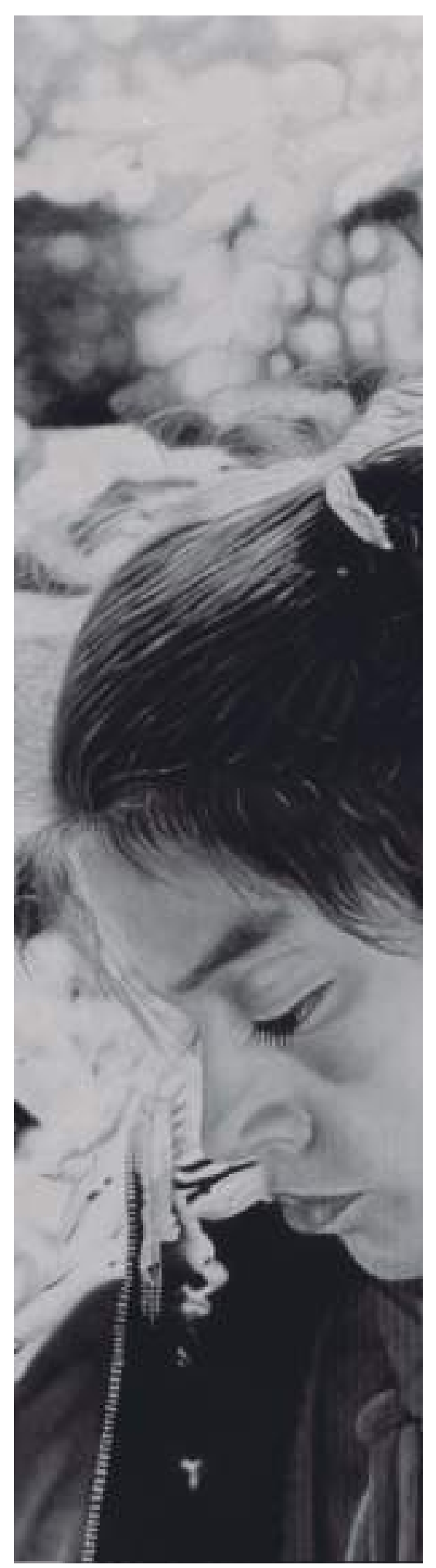




\section{REFERENCIAS}

Arnett, J. (2008). Adolescencia y adultez emergente. Un enfoque cultural. México: Pearson Educación S.A.

Brinkmann, H., Segure, T. y Solar, M. (1989). Adaptación, Estandarización y Elaboración de Normas para el Inventario de Autoestima de Coopersmith. Revista Chilena de Psicología. 10(1), 63-71.

Cué, J. (2014). Cuestionario HONEY-ALONSO. Cuestionario Honey-Alonso de Estilos de Aprendizaje. Recuperado de: http://www.estilosdeaprendizaje.es/chaea/chaea.htm.

García, R. (2011). Estudio sobre la motivación y los problemas de convivencia escolar. (Tesis de maestría). De la base de datos de Repositorio Institucional Universidad de Almería.

González, M. (2011). Estilos de aprendizaje: su influencia para aprender a aprender. Revista de Estilos de Aprendizaje, 7(7), 1-13.

López, J. (1996). Los estilos de aprendizaje y los estilos de enseñanza. Un modelo de categorización de estilos de aprendizaje de los alumnos de enseñanza secundaria desde el punto de vista del profesor. Anales de Psicología, 12(002), 179-184.

Ormrond, J. (2008). Aprendizaje Humano. España, Madrid: Pearson Educación S.A.

Ortíz, J. (2006). Guía Descriptiva para la Elaboración de Protocolos de Investigación. Salud en Tabasco. 12(3), 530-540.

Salas, R. (2008). Estilos de Aprendizaje a la Luz de la Neurociencia. Colombia, Bogotá: Cooperativa Editorial Magisterio.

Pinelo, A. (2008). Estilos de enseñanza de los profesores de la carrera de psicología. Revista Mexicana de Orientación Educativa. 5(13), 17-24.

Woolfolk, A. (2010). Psicología Educativa. México: Pearson Educación S. A.

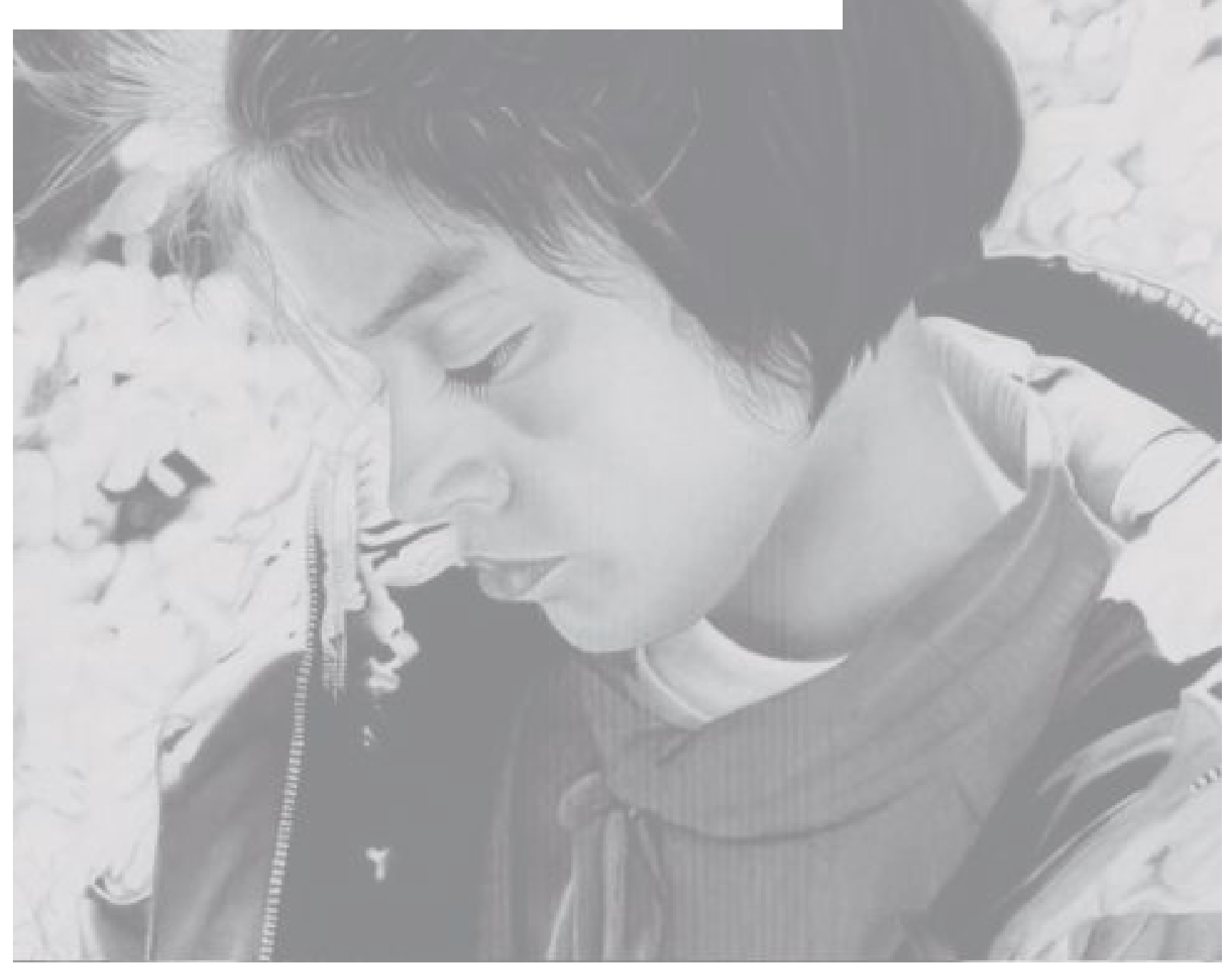




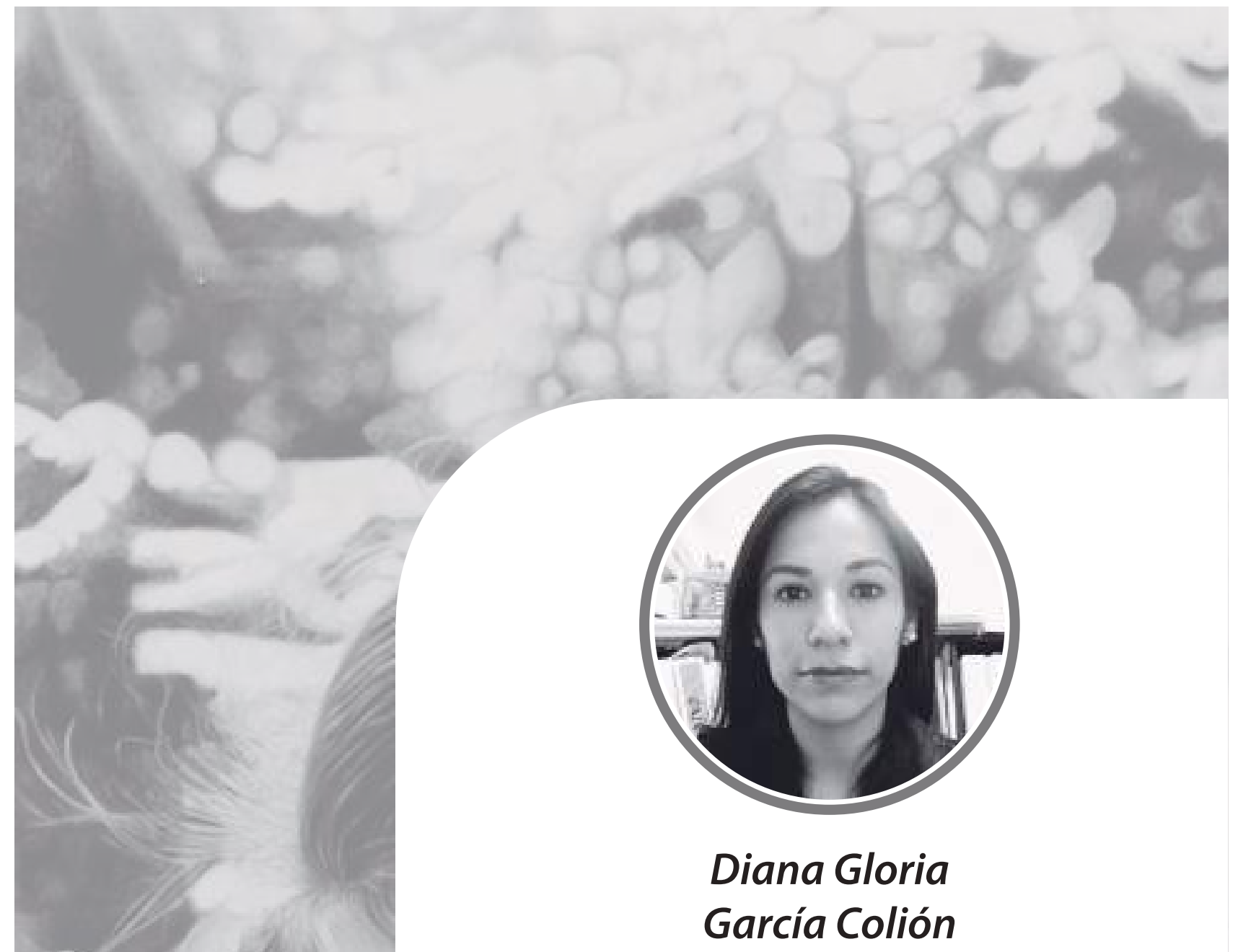

Es Ingeniero en Electrónica y Comunicaciones, egresada del Instituto Tecnológico de Monterrey. En 2014 obtuvo el grado de Maestría en Educación con Mención Honoríica por la misma institución. Se desarrolló en el área de Electrónica y Automatización en TERNIUM por 5 años. Se desempeña como docente de Electrónica en el CBTis74. Es la responsable del programa Construye-T (Desarrollo de Habilidades Socioemocionales) y del programa de Tutorías. Trabaja en conjunto con los maestros del plantel para crear estrategias que permitan a los estudiantes desarrollar habilidades socioemocionales y mejorar su rendimiento académico dándole seguimiento individual.

Correo Electrónico:

dicolion@gmail.com

Recibido: 15/06/2019

Aceptado: 10/09/2019 